\title{
Tissue Plasminogen Activator Induced Delayed Edema in Experimental Porcine Intracranial Hemorrhage: Reduction with Plasminogen Activator Inhibitor-1 Administration
}

\author{
Naureen Keric • Gerrit Steffen Maier • \\ Uzma Samadani • Kai Kallenberg • Peter Dechent • \\ Wolfgang Brueck • Jan Heuer • Veit Rohde
}

Received: 2 February 2012 /Revised: 22 April 2012 / Accepted: 26 April 2012 /Published online: 26 May 2012

(C) Springer Science+Business Media, LLC 2012

\begin{abstract}
Hematoma puncture and subsequent clot lysis with recombinant tissue plasminogen activator (rtPA) emerged as an alternative therapy for spontaneous intracerebral hemorrhage (ICH) and is associated with delayed edema possibly counteracting the beneficial effects of hematoma volume reduction. We hypothesized that immediate reversal of rtPA activity after clot lysis and hematoma drainage diminishes edema formation. To test this hypothesis, we administered plasminogen activator inhibitor (PAI)-1 after rtPA lysis of experimentally induced ICH. A right frontal ICH was placed through a twist drill burr hole and autologous blood injection. Following creation of the frontal ICH, pigs received no further treatment $(n=5)$, lysis with rtPA $(n=7)$, or lysis with rtPA followed by administration of PAI-1 $(n=6)$. Hematoma and edema volumes were assessed with
\end{abstract}

U.S. was supported by the William P. Van Wagenen Fellowship.

N. Keric $\cdot$ G. S. Maier $\cdot$ U. Samadani $\cdot$ V. Rohde

Department of Neurosurgery, Georg-August-University,

Goettingen, Germany

\section{N. Keric}

Department of Neurosurgery,

Johannes-Gutenberg-University,

Mainz, Germany

\section{G. S. Maier}

Department of Orthopedic Surgery,

Johannes-Gutenberg-University,

Mainz, Germany

U. Samadani

Department of Neurosurgery, New York School of Medicine,

New York, NY, USA magnetic resonance imaging on days 0,4 , and 10 . The rtPA significantly reduced hematoma volume and contributed to edema on day 10 after experimentally induced ICH. Administration of PAI-1 attenuated the rtPA-induced edema volume on day 10 , but the hematoma volume reduction was less pronounced. In conclusion, PAI-1 attenuated delayed cerebral edema after rtPA lysis of experimental ICH but also reduced the lytic activity of rtPA. The combination of rtPA clot lysis with PAI-1 might have the potential to further improve the effect of the lytic therapy of ICH, but additional studies to define the optimum time point for PAI-1 administration are required.

Keywords Intracerebral hemorrhage $\cdot$ Pig $\cdot$ Porcine Animal model $\cdot$ Lysis $\cdot$ Recombinant tissue plasminogen activator $\cdot$ Plasminogen activator inhibitor-1
K. Kallenberg $\cdot$ P. Dechent

Department of Neuroradiology, Georg-August University, Goettingen, Germany

W. Brueck

Department of Neuropathology, Georg-August University, Goettingen, Germany

J. Heuer

Department of Anesthesiology, Georg-August University, Goettingen, Germany

V. Rohde $(\bowtie)$

Department of Neurosurgery, University of Goettingen,

Robert-Koch-Str. 40,

37075 Goettingen, Germany

e-mail: veit.rohde@med.uni-goettingen.de 


\section{Introduction}

Spontaneous intracerebral hematomas (ICH) represent approximately $10 \%$ of all strokes and have a 1 -year mortality ranging from 27 to $47 \%$ [1-3]. Prospective studies reveal equally poor outcomes with either surgery or medical management of ICH [4], though a subset of patients with lobar ICH may benefit from the former [5].

Stereotactic puncture and clot aspiration have been considered as an alternative to craniotomy and (micro-) surgical clot removal, with the advantage of avoidance of general anesthesia, decreased surgical time, and diminished operative blood loss [6]. To enhance the amount of hematoma removal, frame-based and frameless stereotactic puncture and aspiration were combined with urokinase or recombinant tissue plasminogen activator (rtPA)-induced clot lysis and subsequent drainage in animal $[7,8]$ and human studies $[9,10]$.

Despite a reduction in hematoma volume, delayed edema formation peaking at day 10 was seen in pigs with rtPAlysed experimental ICH [11]. This edema might limit the benefit of rtPA clot lysis when compared to clot evacuation without lysis [7, 12, 13]. We hypothesized that immediate reversal of rtPA activity after clot lysis and evacuation would diminish such toxicity. To test this hypothesis, we administered plasminogen activator inhibitor (PAI)-1 after rtPA lysis of experimentally induced ICH in a porcine model.

\section{Methods and Materials}

Animals and Surgery

A well-established porcine model of ICH was used for the study [14]. All procedures were performed in accordance with the institutional guidelines for animal welfare and experimental conduct of the University of Göttingen under a protocol approved by the regulatory agencies of Lower Saxony, Germany (33.42502/§9-004/07).

Deutsche Landrasse male pigs of 30-35 kg were sedated with ketamine (10-15 mg/kg IM) and azaperone (7 to $10 \mathrm{mg} / \mathrm{kg}$ ) to allow placement of a venous line. Pentobarbital $15-20 \mathrm{mg} / \mathrm{kg} \mathrm{IV}$, ketamine (1-2 mg/kg IV), and propofol were then administered, and intubation was performed. Respiratory rate and tidal volume were adjusted to maintain physiological end-tidal $\mathrm{CNO}_{2}$ (PEEP, 3-5 mmHg I/E 1:2; TV, $500 \mathrm{ml})$.

A femoral arterial line was placed, and the animal was prepared and draped for surgery. A straight sagittal midline incision was made, and the bregma was exposed. A 3.97-mm twist drill burr hole was placed $0.7 \mathrm{~cm}$ right of midline and $1.7 \mathrm{~cm}$ anterior to the coronal suture. The tip of a 6-French
EMB80 Fogarty balloon catheter (Edwards Lifesciences, Irvine, CA, USA) was introduced to a depth of $1 \mathrm{~cm}$ into the right frontal lobe, and the balloon was inflated for $2 \mathrm{~s}$. A 1.2-cm catheter with an attached reservoir (McCombs catheter, Valley Forge, PA, USA) was then placed into the preformed cavity.

Autologous arterial blood from the femoral line was slowly injected through the reservoir and catheter into the right frontal lobe by using a modified double-injection method ( $2 \mathrm{ml}$ blood, pause of $1 \mathrm{~min}, 5 \mathrm{ml}$ blood) to prevent substantial blood reflux along the catheter into the subdural compartment. Skin closure was performed. The pigs were then taken for MRI under propofol sedation and ventilation with room air.

\section{Magnetic Resonance Imaging}

MRI was performed immediately after surgery (day 0 ) and on days 4 and 10 using a 3.0-T MR imaging system (Magnetom Trio, Siemens Medicals Solutions, Erlangen, Germany) with a standard birdcage head coil. The in-plane resolution was 1.2 by $1.0 \mathrm{~mm}$ for T2*, 1.4 by $1.1 \mathrm{~mm}$ for FLAIR, 1.8 by $1.8 \mathrm{~mm}$ for diffusion, and 0.6 by $0.6 \mathrm{~mm}$ for dual weighted echo. The hematoma volume was calculated on T2* images and the edema volume on FLAIR images [15]. Using the MR imaging software (Syngo, Siemens Medicals Solutions, Erlangen, Germany), the areas of hemorrhage and edema were traced directly on the viewing screen for each slice, and the volume was calculated by a formula of Kothari [16]. The length and equatorial diameter and the number of MRI slices were multiplied and divided by $2(V=A \times B \times C / 2)$ [16].

Fibrinolytic and Plasminogen Activator Inhibitor-1 Therapy

The rtPA (Actilyse, Boehringer Ingelheim, Germany) powder was reconstituted with sterile water to a concentration of $1 \mathrm{mg} / \mathrm{ml}$. Dosage was calculated by multiplying the maximum diameter of the clot (in millimeters) with $0.1 \mathrm{mg}$ rtPA [17]. Immediately after imaging, the rtPA and rtPA-PAI-1 group animals were returned to the operating room for fibrinolytic therapy. The wound was again prepared and draped, and partially reopened to enable clear visualization of the reservoir for rtPA injection. Corresponding to the short half-life of rtPA, the reservoir was re-punctured after $15 \mathrm{~min}$ to aspirate the lysed hematoma. The aspiration was performed carefully over $2 \mathrm{~min}$ in the treated animals but not in the control group.

PAI-1 (Oxford Biomedical Research, Rochester Hills, MI, USA) was resuspended in normal saline to a final concentration of $1.1 \mathrm{mg} / \mathrm{ml}$ and injected into the reservoir directly after aspiration of the hematoma. In all pigs, the reservoir and catheter were left in place, and the wound was again closed. Five pigs, in which only a hematoma was 
created, served as a control group. In seven pigs, fibrinolytic therapy without PAI-1 (rtPA group), and in six pigs, fibrinolytic therapy with additional PAI-1 injection (rtPA-PAI-1 group) were performed.

The hematoma and edema volumes are expressed as mean values $\pm \mathrm{SD}$, and the statistical evaluation was assessed by $t$ test with Statistica software (Statsoft Europe, Hamburg, Germany).

\section{Results}

\section{Hematoma Volume}

The mean hematoma volume was $3.84 \mathrm{ml}( \pm 2.4 \mathrm{ml})$ in the control group, $3.7 \mathrm{ml}( \pm 2.65 \mathrm{ml})$ in the rtPA group, and $3.5 \mathrm{ml}( \pm 2.65 \mathrm{ml})$ in the rtPA-PAI-1 group directly after surgery. On day 4 , the mean hematoma volume dropped to $3.01 \mathrm{ml}( \pm 2.02 \mathrm{ml})$ in the control group, to $2.42 \mathrm{ml}$ $( \pm 2.02 \mathrm{ml})$ in the rtPA group, and to $2.46 \mathrm{ml}( \pm 1.28 \mathrm{ml})$ in the rtPA-PAI-1group. On day 10, a further reduction of the hematoma volume could be seen. The hematoma volume was $2.62 \mathrm{ml}( \pm 0.87 \mathrm{ml})$ in the control group, $1.36 \mathrm{ml}$ $( \pm 1.12 \mathrm{ml})$ in the $\mathrm{rtPA}$ group, and $2.12 \mathrm{ml}( \pm 0.98 \mathrm{ml})$ in the rtPA-PAI-1 group.

The mean volume reduced by aspiration $15 \mathrm{~min}$ after lysis was $0.97 \mathrm{ml}( \pm 1 \mathrm{ml})$ in the rtPA group and $1.2 \mathrm{ml}$ $( \pm 1.5 \mathrm{ml})$ in the rtPA-PAI-1 group. The overall hematoma volume reduction was $31.8 \%$ in the control group, $63.3 \%$ in the rtPA group, and $39.5 \%$ in the rtPA-PAI- 1 group. Compared to the control group, the hematoma volume reduction was significantly higher $(p=0.034)$ in the rtPA group, whereas the hematoma volume reduction in the rtPA-PAI-1 group showed no significant difference (Fig. 1).

\section{Cerebral Edema}

The mean edema volume on day 0 was $0.91 \mathrm{ml}( \pm 2.82 \mathrm{ml})$ in the control group, $0.53 \mathrm{ml}( \pm 0.62 \mathrm{ml})$ in the rtPA group, and $2.46 \mathrm{ml}( \pm 3.72 \mathrm{ml})$ in the rtPA-PAI-1 group. On day 4 , an edema volume of $14.82 \mathrm{ml}( \pm 9.45 \mathrm{ml})$ in the control group, $17.37 \mathrm{ml}( \pm 8.2 \mathrm{ml})$ in the rtPA group, and $24.62 \mathrm{ml}( \pm 11.21 \mathrm{ml})$ in the rtPA-PAI-1 group was measured. On day 10, the edema volume had dropped to $12.9 \mathrm{ml}( \pm 11.15 \mathrm{ml})$ in the control group and to $11.94 \mathrm{ml}( \pm 11.93 \mathrm{ml})$ in the rtPA-PAI-1 group, whereas in the rtPA group, a further increase to $30.94 \mathrm{ml}$ $( \pm 16.02 \mathrm{ml})$ was seen.

The overall edema volume increase was $1,411 \%$ in the control group, $5,937 \%$ in the rtPA group, and $485 \%$ in the rtPA-PAI-1 group. On day 4, differences in the amount of edema were not significant among the treatment groups. Student's $t$ test demonstrated significant differences in edema volume on day 10 between the control and the rtPA group $(p=0.05)$ and again at day 10 between the rtPA and the rtPA-PAI-1 groups ( $p=0.032$ ) (Fig. 2).

\section{Discussion}

Clinical series have demonstrated that fibrinolytic therapy after stereotactic puncture allows reducing the volume of a spontaneous ICH by 60 to $70 \%$. In most of these clinical studies, the mortality rates had been lower in comparison to a series of (micro-)surgical clot removal or best medical therapy, which was believed to be the result of effective volume
Fig. 1 Mean hematoma volume of each group at day 0 and 10 with $S D$ bars. There is a significant decrease in hematoma volume in the rtPA group $(n=7)$ compared to the control group $(n=5)$. The rtPA-PAI-1 group $(n=6)$ shows a not significant decrease of hematoma volume

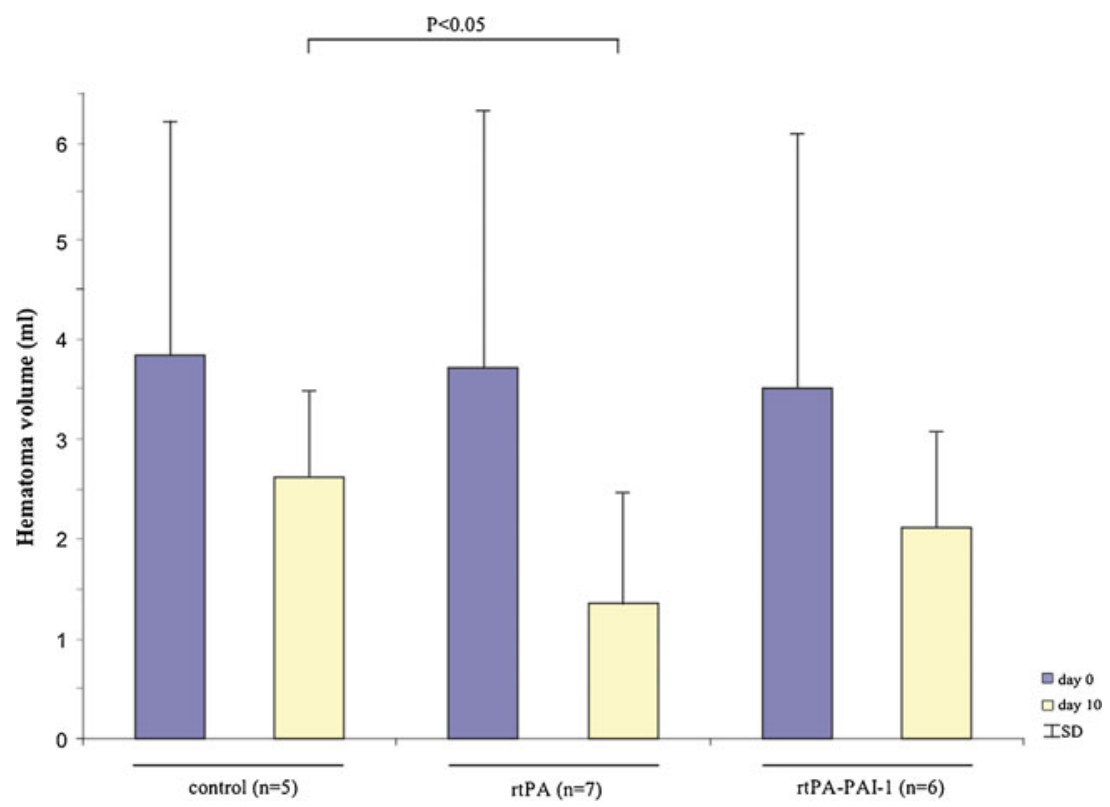




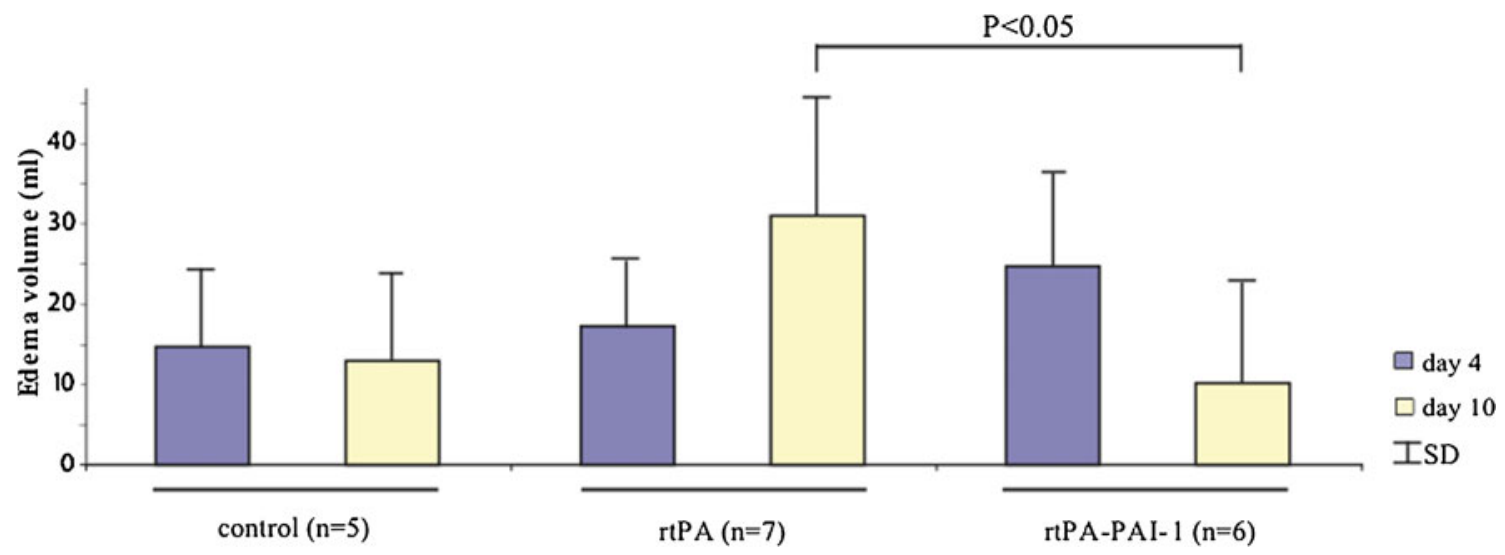

Fig. 2 Mean edema volumes of each group are shown at day 4 and 10 with SD bars. A significant difference was seen between the rtPA $(n=7)$ and rtPA-PAI-1 group $(n=6)$

reduction despite the minimal invasiveness of the procedure. However, in the only available randomized trial, comparing fibrinolytic therapy with conservative management, a positive effect of stereotaxy and clot lysis on outcome could not been demonstrated [18]. Animal experiments have indicated that rtPA is effective in reducing the hematoma volume but is accompanied by a delayed perihematomal edema [19-22]. It seems possible that the occurrence of the delayed edema attenuates or eliminates the positive effect of accelerated clot lysis by rtPA on outcome and may explain the equivocal clinical results [11].

\section{Perihematomal Edema}

The present experimental study confirms again the observations that fibrinolytic therapy in experimental ICH induces delayed perihematomal edema. There are several explanations for the occurrence of the delayed edema. (1) In vitro mouse neuronal cultures showed that endogenous tPA mediates microglial activation and excitotoxic neuronal degeneration $[23,24]$. In line with this finding, mice deficient in tPA have reduced edema compared to wild-type mice after brain injury and focal cerebral ischemia [25]. As hypoperfusion

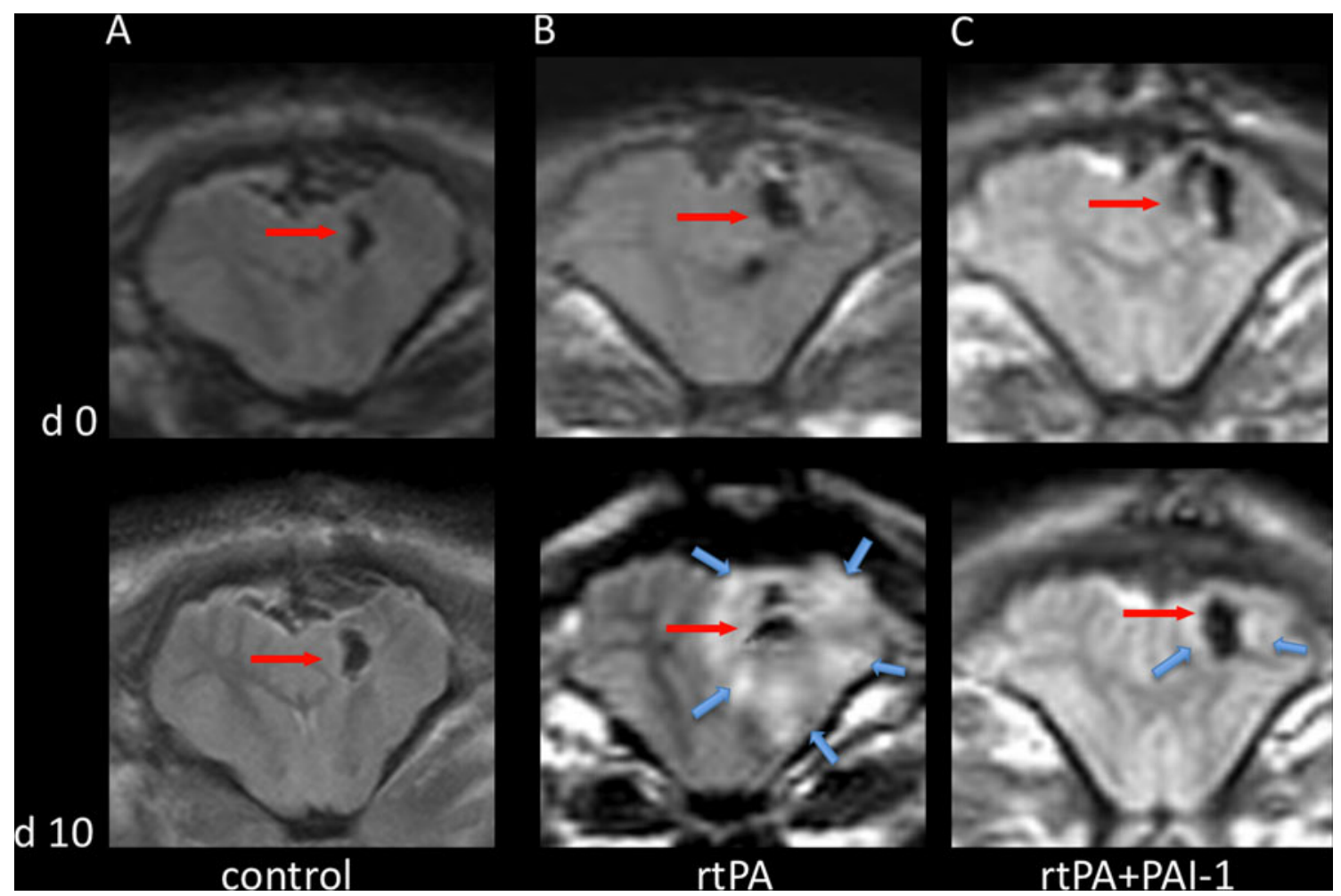

Fig. 3 Coronal MR-FLAIR images of one animal in each group $(\mathbf{a}, \mathbf{b}, \mathbf{c})$ in the upper lane immediately after surgery at day 0 and in the lower lane at day 10. The red arrows indicate $\mathrm{ICH}$, and the blue arrows indicate edema 
and ischemia occur in the penumbra of the intracerebral hematoma, adding exogenous tPA as in fibrinolytic therapy for ICH probably enhances these processes leading to edema formation. Accordingly, we have been able to show in a recent animal experiment that the late edema after experimental clot lysis could be reduced using MK 801 for blockage of the excitotoxicity-mediating NMDA receptors [26]. (2) Furthermore, at least one study showed that direct injection of rtPA into the rat brain is neurotoxic [21], possibly partly mediated via compromise of the blood-brain barrier [27]. (3) Thrombin, which is inhibited by PAI-1, is one of the major sources of perihematomal edema [28]. The intracerebral concentration of PAI-1 after experimental ICH in rats is significantly higher in the perihematomal brain tissue compared to the contralateral hemisphere [29], possibly indicating that the attempt is made to control the increased thrombin release after ICH. The rtPA likewise is inhibited by PAI-1. If not compensated by the upregulation of endogenous PAI-1, the administration of rtPA during fibrinolytic therapy reduces the relative concentration of PAI-1 available for thrombin inactivation, thus leading to edema.

\section{Rationale for PAI-1 Therapy}

The occurrence of a delayed perihematomal edema after fibrinolytic therapy, as seen in our previous and this study, indicates that the increase of endogenous PAI-1 fails to prevent higher thrombin concentrations. Therefore, we hypothesized that exogenous PAI-1, given after tPA-lysis, attenuates edema formation by inhibiting both thrombin and exogenous rtPA. The finding of the study proved the hypothesis to be correct, as the perihematomal edema was significantly lower in the rtPA-PAI-1 than in the rtPA group. Furthermore, administration of a hexapeptide mimicking the action of PAI-1 was neuroprotective in rat stroke models [30]. In vitro experiments in neural differentiated pheochromocytoma cells indicated an additional anti-apoptotic effect of PAI-1 [31]. Possibly, these direct neuroprotective effects of PAI-1 contributed to the documented edema reduction.

\section{Clot Lysis and PAI-1}

The present experiment showed that injection of PAI-1 after rtPA clot lysis and aspiration of the liquefied blood attenuates not only formation of the delayed edema but also reduction of the clot volume. The positive effect of edema volume reduction might be decreased by less effective hematoma volume reduction. Hematoma and edema volume reduction is necessary for optimal clinical outcome. In this setting, the timing of rtPA administration and clot aspiration was corresponded to its short half time of about $20 \mathrm{~min}$. Expecting a sufficient clot reduction after lysis and aspiration, PAI-1 was administered immediately after clot aspiration. In contrast to our previous findings [7], there is a progressive decrease of the hematoma volume from day 0 to 10 in the rtPA group (Fig. 3). Probably, this suggests an ongoing clot lysis after aspiration or could also be drainage of liquefied clot into the subdural space. An ongoing effect might be interrupted by the administration of PAI-1. Future experimental studies should focus on definition of the optimum PAI-1 administration time point to achieve both maximum clot lysis by rtPA and avoidance of edema formation. If this time point is identified, the combination of rtPA and PAI-1 compared to single rtPA in the fibrinolytic therapy of intracerebral hemorrhage has the potential to improve the clinical results.

\section{Conclusion}

PAI-1 not only diminishes cerebral edema after rtPA treatment of experimental ICH in pigs but also attenuates the hematoma lysis by rtPA. We propose that the time point of administration of PAI-1 after rtPA lysis needs further investigation and can lead to a more neuroprotective and effective rtPA therapy of ICHs.

Conflict of interest We state that the study represents the authors' own work. Author Uzma Samadani received financial support in favor of this study from the William P. Van Wagenen Fellowship Programme. All authors declare that they have no conflict of interest. We would like to further state that animal experiments were strictly adherent to the institutional guidelines for animal welfare and experimental conduct of the University of Göttingen under a protocol approved by the regulatory agencies of Lower Saxony, Germany (33.42502/§9-004/07).

\section{References}

1. Hanel RA, Xavier AR, Mohammad Y, Kirmani JF, Yahia AM, Qureshi AI. Outcome following intracerebral hemorrhage and subarachnoid hemorrhage. Neurol Res. 2002;24(1):58-62.

2. Nilsson OG, Lindgren A, Brandt L, Saveland H. Prediction of death in patients with primary intracerebral hemorrhage: a prospective study of a defined population. J Neurosurg. 2002;97:5316.

3. Schutz H, Bodeker RH, Damian M, Krack P, Dorndorf W. Agerelated spontaneous intracerebral hematoma in a German community. Stroke. 1990;21:1412-8.

4. Manno EM, Atkinson JL, Fulgham JR, Wijdicks EF. Emerging medical and surgical management strategies in the evaluation and treatment of intracerebral hemorrhage. Mayo Clin Proc. 2005;80:420-33.

5. Hardemark HG, Wesslen N, Persson L. Influence of clinical factors, CT findings and early management on outcome in supratentorial intracerebral hemorrhage. Cerebrovasc Dis. 1999;9:10 21.

6. Carvi y Nievas MN, Haas E, Hollerhage HG, Schneider H, Pollath A, Archavlis E. Combined minimal invasive techniques in deep supratentorial intracerebral haematomas. Minim Invasive Neurosurg. 2004;47:294-8. 
7. Thiex R, Kuker W, Muller HD, Rohde I, Schroder JM, Gilsbach JM, Rohde $\mathrm{V}$. The long-term effect of recombinant tissue-plasminogenactivator (rt-PA) on edema formation in a large-animal model of intracerebral hemorrhage. Neurol Res. 2003;25:254-62.

8. Wagner KR, Xi Guohua Y, Zuccarello M, de Courten-Myers GM, Broderick JP, et al. Ultra-early clot aspiration after lysis with tissue plasminogen activator in a porcine model of intracerebral hemorrhage: edema reduction and blood-brain barrier protection. J Neurosurg. 1999;90:491-8.

9. Rohde V, Uzma N, Rohde I, St Clair E, Samadani U. Fibrinolytic therapy versus craniotomy for anticoagulant-associated intracerebral hemorrhage. Clin Neurol Neurosurg. 2009;111(6):518-22.

10. Rohde V, Schaller C, Hassler WE. Intraventricular recombinant tisuue plasminogen activator for lysis of intraventricular hemorrhage. J Neurol Neurosurg Psychiatry. 1995;58:447-51.

11. Rohde V, Rohde I, Thiex R, Ince A, Jung A, Duckers G, et al. Fibrinolysis therapy achieved with tissue plasminogen activator and aspiration of the liquefied clot after experimental intracerebral hemorrhage: rapid reduction in hematoma volume but intensification of delayed edema formation. J Neurosurg. 2002;97:954-62.

12. Hoff JT, Xi G. Brain edema from intracerebral hemorrhage. Acta Neurochir. 2003;86:11-5.

13. Thiex R, Kuker W, Jungbluth P, Kayser C, Muller HD, Rohde I, Gilsbach JM, Rohde V. Minor inflammation after surgical evacuation compared with fibrinolytic therapy of experimental intracerebral hemorrhages. Neurol Res. 2005;27:493-8.

14. Wagner KR, Xi G, Hua Y, Kleinholz M, de Courten-Myers GM, Myers RE, et al. Lobar intracerebral hemorrhage model in pigs: rapid edema development in perihematomal white matter. Stroke. 1996;27:490-7.

15. Tsuchiya K, Mizutani Y, Hachiya J. Preliminary evaluation of fluid-attenuated inversion-recovery MR in the diagnosis of intracranial tumors. Am J Neuroradiol. 1996;17(6):1081-6.

16. Kothari R, Pancioli A, Brott T, Broderick J. Thrombolytic therapy for cerebral infarction. Acad Emerg Med. 1996;3(9):881-92.

17. Schaller C, Rohde V, Meyer B, Hassler W. Stereotactic puncture and lysis of spontaneous intracerebral hemorrhage using recombinant tissue-plasminogen activator. Neurosurgery. 1995;36(2):328 33 .

18. Teernstra OP, Evers SM, Lodder J, Leffers P, Franke CL, Blaauw G. Stereotactic treatment of intracerebral hematoma by means of a plasminogen activator: a multicenter randomized controlled trial (SICHPA). Stroke. 2003;34(4):968-74.
19. Tsirka SE. Clinical implications of the involvement of tPA in neuronal cell death. J Mol Med. 1997;75(5):341-7.

20. Figueroa BE, Keep RF, Betz AL, Hoff JT. Plasminogen activators potentiate thrombin-induced brain injury. Stroke. 1998;29(6):12027.

21. Wang X, Asahi M, Lo EH. Tissue type plasminogen activator amplifies hemoglobin-induced neurotoxicity in rat neuronal cultures. Neurosci Lett. 1999;274(2):79-82.

22. Goto H, Fujisawa H, Oka F, Nomura S, Kajiwara K, Kato S, Fujii M, Maekawa T, Suzuki M. Neurotoxic effects of exogenous recombinant tissue-type plasminogen activator on the normal rat brain. J Neurotrauma. 2007;24:745-52.

23. Rogove AD, Siao C, Keyt B, Strickland S, Tsirka SE. Activation of microglia reveals a non-proteolytic cytokine function for tissue plasminogen activator in the central nervous system. J Cell Sci. 1999;112:4007-16.

24. Siao CJ, Tsirka SE. Tissue plasminogen activator mediates microglial activation via its finger domain through annexin II. J Neurosci. 2002;22:3352-8.

25. Mori T, Wang X, Kline AE, Siao CJ, Dixon CE, Tsirka SE, et al. Reduced cortical injury and edema in tissue plasminogen activator knockout mice after brain trauma. Neuroreport. 2001;12:4117-20.

26. Thiex R, Weis J, Krings T, Barreiro S, Yakisikli-Alemi F, Gilsbach JM, et al. Addition of intravenous N-methyl-D-aspartate receptor antagonists to local fibrinolytic therapy for the optimal treatment of experimental intracerebral hemorrhages. J Neurosurg. 2007;106:314-20.

27. Yepes M, Sandkvist M, Moore EG, Bugge TH, Strickland DK, Lawrence DA. Tissue-type plasminogen activator induces opening of the blood-brain barrier via the LDL receptor-related protein. J Clin Invest. 2003;112:1533-40.

28. Chen B, Cheng Q, Yang K, Lyden PD. Thrombin mediates severe neurovascular injury during ischemia. Stroke. 2010;41(10):2348 52. Epub 2010 Aug 12.

29. Hua Y, Xi G, Keep RF, Wu J, Jiang Y, Hoff JT. Plasminogen activator inhibitor-1 induction after experimental intracerebral hemorrhage. J Cereb Blood Flow Metab. 2002;22:55-61.

30. Armstead WM, Nassar T, Akkawi S, Smith DH, Chen XH, Cines DB, et al. Neutralizing the neurotoxic effects of exogenous and endogenous tPA. Nat Neurosci. 2006;9:1150-5.

31. Soeda S, Koyanagi S, Kuramoto Y, Kimura M, Oda M, Kozako T, et al. Anti-apoptotic roles of plasminogen activator inhibitor-1 as a neurotrophic factor in the central nervous system. Thromb Haemost. 2008;100(6):1014-20. Review. 